\title{
Communication
}

\section{Detection and sequencing of new cyclic peptides from linseed by electrospray ionization mass spectrometry ${ }^{\star \star}$}

\author{
Piotr Stefanowicz ${ }^{\bowtie}$
}

Faculty of Chemistry, University of Wrocław, Wroctaw, Poland

Received: 19 September, 2001; accepted: 26 November, 2001

Key words: cyclolinopeptides, mass spectrometry

\begin{abstract}
Extract of Linum usitatissimum seeds was analyzed by ESI-MS and ESI-MS/MS. The analysis confirms the presence of previously reported cyclolinopeptides: CLA (c(ProPro-Phe-Phe-Leu-Ile-Ile-Leu-Val) and CLB (c(Pro-Pro-Phe-Phe-Val-Ile-Met-Ile-Leu)). Cyclolinopeptides CLD and CLE, which contain methionine oxide, were detected in small quantities only. These peptides likely result from the oxidation of their precursors, not reported previously: CLD' (c(Pro-Phe-Phe-Trp-Ile-Met-Leu-Leu)) and CLE'(c(Pro-Leu-Phe-Ile-Met-Leu-Val-Phe)), present at higher concentrations in unoxidized extract. Two new cyclic octapeptides: CLF (c(Pro-Phe-Phe-Trp-Val-Met-LeuMet)) and CLG (c(Pro-Phe-Phe-Trp-Ile-Met-Leu-Met)) were detected and their sequences were proposed on the basis of CID experiments and similarity with those of CLD'.
\end{abstract}

Cyclolinopeptides - cyclic, hydrophobic peptides isolated from linseed exhibit various biological activities. Cyclolinopeptide A and its synthetic analogues have immunosuppressive activity [1]. The mechanism of immunosuppression caused by CLA and its analogues is similar to that of cyclosporine [2]. There are also reports on immunosuppressive properties of cyclolinopeptides B and E [3]. CLA exhibits hepatoprotective properties in the causes of poisoning by Amanita phaloides toxins [4]. Recent data indicate antimalarial activity of CLA [5]. Cyclolinopeptide A, one of the earliest disovered naturally occurring cy-

\footnotetext{
${ }^{\star}$ Presented at the XVI Polish Peptide Symposium, September 1-4, 2001, Jagiellonian University, Kraków, Poland.

This work was supported by the State Committee for Scientific Research (KBN, Poland) grant No. 3T09A 03618.

${ }^{\otimes}$ Corresponding author: Piotr Stefanowicz, Wydział Chemii, Uniwersytet Wrocławski, F. Joliot-Curie 14, 50-383 Wrocław, Poland; tel: (48 71) 375 7213; e-mail: stp@wchuwr.chem.uni.wroc.pl

Abbreviations: CID, collision induced dissociation; ESI-MS, electrospray ionization mass spectrometry; ESI-MS/MS, electrospray ionization tandem mass spectrometry.
} 
clic peptides has been known since 1959 [6], $\mathrm{CLB}$, the another representative of this group of compounds was reported in 1968 [7].

In a recent paper, Hiroshi Morita et al. [3] reported on a series of new cyclic peptides isolated from linseed. These peptides were isolated from $30 \mathrm{~kg}$ of seeds of Linum usitatissimum and purified by chromatography. Their structures have been elucidated on the basis of 2D NMR and chemical degradation.

In our studies the ESI-MS and ESI-MS/MS techniques were applied for the characterization of linseed extract confirming presence of cyclolinopeptides CLA - CLE. Two new analogues CLF and CLG were found. Because the MS based procedure is much faster than NMR and does not require highly purified material, detection of minor or unstable peptides was possible. The high sensitivity of the mass spectrometric method is also an advantage.

\section{MATERIALS AND METHODS}

Extraction of peptides. Five g of ground linseed was extracted with $100 \mathrm{ml}$ of acetone at room temperature overnight. After concentration in vacuo the mixture was dissolved in methanol and hydrolyzed with $10 \% \mathrm{NaOH}$. After evaporation under reduced pressure, the fraction containing peptides was isolated by ethyl acetate extraction, evaporated and used in ESI-MS experiments without further purification.

ESI experiments. A Finnigan MAT TSQ -700 mass spectrometer equipped with ESI source was used. Samples were dissolved in methanol containing $10 \mathrm{mM}$ of ammonium acetate. Fragmentation experiments were performed by the MS/MS technique.

\section{RESULTS AND DISCUSSION}

Crude, peptide fraction of linseed was studied by ESI-MS. The applied method of extraction removes all linear peptides present in the sample. Also cyclic peptides containing Asp or Glu residues would be removed because of the basic $\mathrm{pH}$ during extraction.

High signal intensity of the cyclic peptides was achieved by: using of $10^{-4} \mathrm{M}$ solution of $\mathrm{NaCl}$ in $\mathrm{MeOH}$ or $10^{-2} \mathrm{M}$ solution of ammonium acetate in $\mathrm{MeOH}$ as solvent for the samples. In the first case metalated ions of $[\mathrm{MNa}]^{+}$type are observed, while the second approach yields $[\mathrm{MH}]^{+}$ions. A spectrum of peptide fraction of linseed extract ionized by protonation is presented in Fig. 1. The molec-

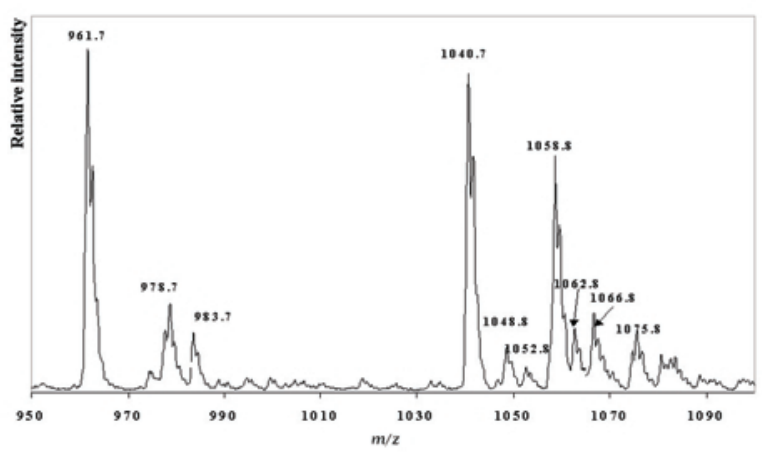

Figure 1. ESI spectrum of peptide fraction of linseed.

Sample, prepared according to Materials and Methods, dissolved in $10 \mathrm{mM}$ ammonium acetate in methanol was infused into the ion source at a flow rate $2 \mu \mathrm{l} / \mathrm{min}$.

ular masses of the compounds observed presented in Table 1 were compared with the masses of the peptides detected in linseed by Hiroshi Morita et al. [3]. The data presented in Table 1 show that many detected compounds correspond to the already reported cyclolinopeptides. On the other hand, certain masses do not correspond to the reported peptides, which may indicate the presence of the novel compounds. Peptides with molecular masses not reported in the literature were subjected to CID experiment, which was performed on protonated ions $[\mathrm{MH}]^{+}$.

The masses 961.8 and 1048.8 correspond to unoxidized forms of the known peptides and CLE and CLD, respectively. In order to confirm this hypothesis CID spectra of peptides with molecular masses 1048.8 and 961.8 were 
Table 1

\begin{tabular}{lll}
\hline Mass of protonated ion $[\mathrm{MH}]^{+}$ & Sequences for reported peptides & $\begin{array}{l}\text { Symbol of peptide according to } \\
\text { Morita et al. [3] }\end{array}$ \\
\hline 961.8 & & $\left(\mathrm{CLE}^{\prime}\right)^{*}$ \\
977.6 & c(PLFIMOLVF) & CLE \\
1040.8 & c(PPFFLIILV) & CLA \\
1048.8 & & $(\mathrm{CLD})^{*}$ \\
1052.6 & & $(\mathrm{CLF})^{*}$ \\
1058.6 & c(PPFFVIMIL) & CLB \\
1064.8 & c(PFFWIMOLL) & CLD \\
1066.8 & & (CLG)* \\
1074.9 & c(PFFWVMOLL) & CLC* \\
1082.8 & & \\
\hline
\end{tabular}

*Abbreviations proposed in this paper

obtained. Figure $2 \mathrm{~A}$ and $2 \mathrm{~B}$ shows the results of the fragmentation of these peptides. In both cases one predominant series of product ions of type B is observed. Data presented in the spectrum $2 \mathrm{~A}$ show clearly that the studied peptide with $\mathrm{M} 1048.8\left(\mathrm{CLD}^{\prime}\right)$ is the unoxidized form of the known peptide CLD.

A

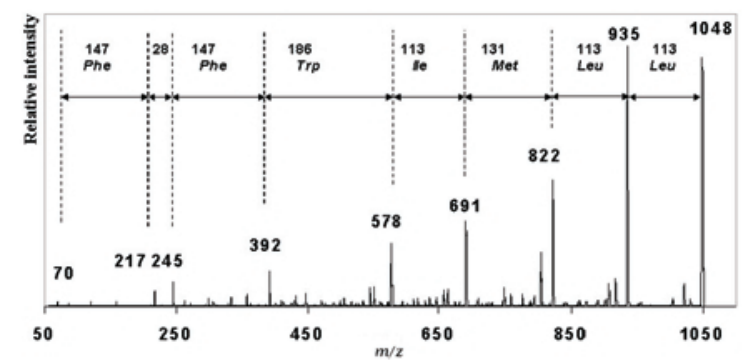

tons and determine the location of charge in a cyclic peptide [8]. The fragmentation pattern for the peptide with $[\mathrm{MH}]^{+} 961.8$ is more complicated, because besides the main ring opening process (between Pro and Phe) less abundant fragment ions corresponding to opening of the peptide ring between Phe and Val, Val

B

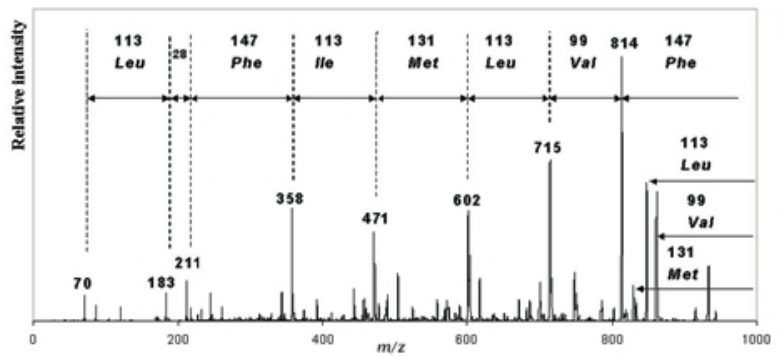

Figure 2. Product ion spectra of the ion at $m / z 1048.8$ (panel A, peptide $C L D^{\prime}$ ) and the ion at $m / z 961.8$ (panel B, peptide $\mathrm{CLE}^{\prime}$ ).

The general scheme of fragmentation of this peptide is consistent with that observed for synthetic analogues of CLA. Ring opening of the cyclic peptide takes place at the amide nitrogen of proline, and gradual neutral losses give rise to a series of fragment ions of the $B$ type. The presented mechanism of fragmentation is typical for cyclic peptides containing proline or thiazolodinecarboxylic acid. These amino acids have the highest affinity for pro- and Leu/Ile, and Leu/Ile and Met are also observed. These additional cleavage processes do not complicate the interpretation of the data because of the relatively low abundance of the corresponding peaks. The presented results confirmed the sequence proposed for this peptide $\left(\mathrm{CLE}^{\prime}\right)$, which is the unoxidized form of the peptide CLE. This interpretation found additional support in an MS/MS/MS experiment (not shown). The assumption, 
that detected peptides are cyclic is additionally supported by two facts:

1. Molecular mass calculated by adding molecular masses of all the amino-acid residues identified in a peptide is higher by $18 \mathrm{Da}$ than the molecular mass measured experimentally.

For peptides containing only Leu, Ile, Phe, Val, Met, Pro and Trp residues, cyclization is the most likely modification process, explaining the loss of the 18 Da.

2 . In some cases parallel elimination of several amino acid-residues was observed. For example the molecular ion of $\mathrm{CLE}^{\prime}$ may eliminate Leu/Ile or Val or Met. Such a combination of neutral losses is highly unlikely for linear peptides.

The masses 1052.8 and 1066.8 correspond to novel peptides. A significant number of fragment ions of peptides at $\mathrm{m} / z 1052.8$ and 1066.8 (Fig. 3) are exactly the same as fragment ions of CLD', which is the unoxidized form of peptide CLD. An analysis of fragmen-

A

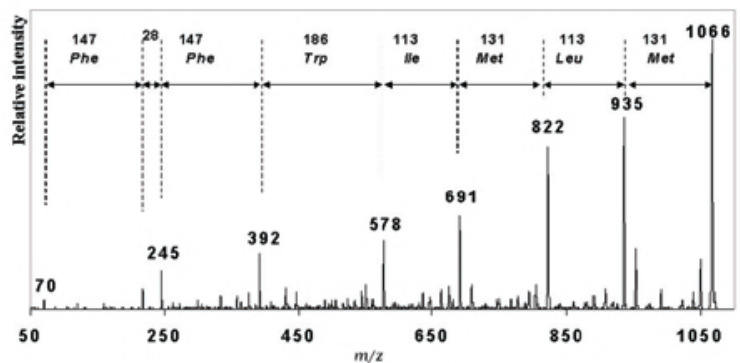

cluded. Because all known peptides isolated from linseed contain exclusively L-amino acids, the assumption that new compounds are also composed of L-amino acids only, seems well justified.

ESI-MS and ESI-MS/MS provided data which would be difficult to obtain by the NMR, because of the low concentration and unstability of certain compounds. The method used here requires about $10^{4}$ times less raw material then NMR and all experiments, including the extraction take only several days. On the other hand, the basic limitation of the mass spectrometric procedure is its inability to determine the configuration of amino-acid residues and difficulties with distinguishing between leucine and isoleucine. Because the analysis performed produced only a few possible sequences for the novel peptides, two in first case and four in the other, synthesis of all possible analogues needed for biological tests is a faster and less expensive method than isolation of particular components and determi-

B

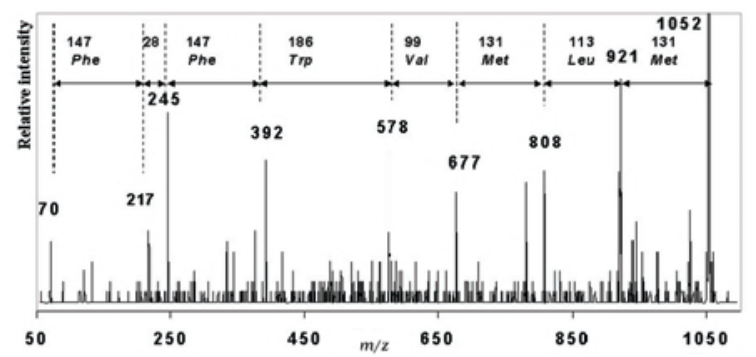

Figure 3. Product ion spectra of the ion at $m / z 1066.8$ (panel A, peptide CLG) and the ion at $m / z 1052.6$ (panel B, peptide CLF).

tations reveals that there is a close homology among all these peptides. On the basis of a comparison of the fragmentation of CLD and the peptides characterized by $[\mathrm{MH}]^{+} 1052.8$ and 1066.8 the sequences c(Pro-Phe-Phe-TrpVal-Met-Leu/Ile-Met) and c(Pro-Phe-Phe-TrpLeu/Ile-Met-Leu/Ile-Met), respectively, were proposed. The most likely positions of Leu and Ile were assigned on the basis of homology with the reported peptide CLD, however other combinations can not be ex- nation of their structures by more rigorous methods.

Mass spectrometric analysis of the composition of an extract without a separation step often results in losses of certain peaks. Parallel experiment performed on fractions separated off line did not show any peptide which was not detected in the crude mixture. However, this result is not general, and direct MS analysis is possible only for mixtures containing a limited number of components. 


\section{CONCLUSIONS}

Our experiments confirm the structures of the peptides reported by Morita et al. [3] and show the presence of two novel cyclolinopeptides CLF (c(Pro-Phe-Phe-Trp-Val-Met-LeuMet)) and CLG (c(Pro-Phe-Phe-Trp-Ile-MetLeu-Met)). The sequences of these peptides were established on the basis of their CID spectra and homology with CLD. We also show, that freshly extracted material contains no or little oxidized peptides. Forms containing methionine oxide likely result from extract oxidation by air. The quick oxidation of methionine containing peptides may be a result of a radical process connected to oxidation of polyunsaturated acids present in linseed oil [9] but confirmation of this hypothesis needs further experiments. The presented method is much faster and less expensive than NMR based procedures.

\section{R E F E R E N C E S}

1. Wieczorek, Z., Bengtson, B., Trojnar, J. \& Siemion, I.Z. (1991) Peptide Res. 4, 275-283.
2. Gaymes, T.J., Cebrat, M., Siemion, I.Z. \& Kay, J.E. (1997) FEBS Lett. 418, 224-227.

3. Morita, H., Shishido, A., Matsumoto, T., Itokawa, H. \& Takeya, K. (1999) Tetrahedron 55, 967-976.

4 Kessler, H., Heupt, M., Frimmer, K. \& Zigler, K. (1986) Int. J. Peptide Protein Res. 29, 621-628.

5. Bell, A., McSteen, P.M., Cebrat, M., Picur, B. \& Siemion, I.Z. (2000) Acta Pol. Pharm. 57, (Suppl.) 134-136.

6. Kaufmann, H.P. \& Tobschirbel, A. (1959) Chem. Ber. 92, 2805-2809.

7. Weygand, F. (1968) Z. Anal. Chem. 243, 2.

8. Eckart, K. (1994) Mass Spectrom. Rev. 13, 23-55.

9. Nielsen, H.K., Loliger., J. \& Hurrell, R.F. (1985) Br. J. Nutr. 53, 61-73. 